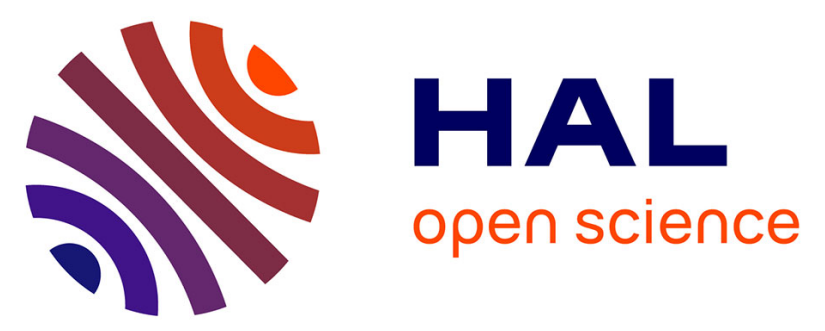

\title{
Experimental use of photodynamic therapy in high grade gliomas: a review focused on 5-aminolevulinic acid.
}

\author{
Marie-Charlotte Tetard, Maximilien Vermandel, Serge Mordon, Jean-Paul
}

Lejeune, Nicolas Reyns

\section{- To cite this version:}

Marie-Charlotte Tetard, Maximilien Vermandel, Serge Mordon, Jean-Paul Lejeune, Nicolas Reyns. Experimental use of photodynamic therapy in high grade gliomas: a review focused on 5-aminolevulinic acid.. Photodiagnosis and Photodynamic Therapy, 2014, pp.319-30. hal-01181357

\section{HAL Id: hal-01181357 \\ https://hal.science/hal-01181357}

Submitted on 30 Jul 2015

HAL is a multi-disciplinary open access archive for the deposit and dissemination of scientific research documents, whether they are published or not. The documents may come from teaching and research institutions in France or abroad, or from public or private research centers.
L'archive ouverte pluridisciplinaire HAL, est destinée au dépôt et à la diffusion de documents scientifiques de niveau recherche, publiés ou non, émanant des établissements d'enseignement et de recherche français ou étrangers, des laboratoires publics ou privés. 
Experimental use of Photodynamic Therapy in high grade
gliomas: a review focused on 5 -aminolevulinic acid.

Marie-Charlotte Tetard $1,2,3$, MD, Maximilien Vermandel1,2,3, PhD, Serge Mordon³, PhD, JeanPaul Lejeune ${ }^{1,2,3}$, MD, Nicolas Reyns $1,2,3$, MD, PhD

1- University hospital of Lille - CHRU, Lille F-59000, France

2 - Université de Lille 2, Lille, F59000, France

3 - Inserm, U703 - ThIAIS, Loos, F59120, France

Keywords: 5-aminolevulinic acid (ALA), Photodynamic therapy, Brain tumour, High-Grade Glioma, preclinical model.

* Corresponding Author: m-vermandel@chru-lille.fr

\begin{abstract}
:
Photodynamic therapy (PDT) consists of a laser light exposure of tumour cells photosensitized by general or local administration of a pharmacological agent. Nowadays, PDT is a clinically established modality for treatment of many cancers consisting.

5-aminolevulinic acid (ALA) induced Protoporphyrin IX (PpIX) has proven its rational in fluoro-guided resection of malignant gliomas due to a selective tumour uptake and minimal skin sensitization. Moreover, the relatively specific accumulation of photosensitizing PPIX within the tumour cells has gained interest in the PDT of malignant gliomas. Several experimental and clinical studies have then established ALA-PDT as a valuable adjuvant therapy in the management of malignant gliomas. However, the procedure still requires optimizations in the fields of tissue oxygenation status, photosensitizer concentration or scheme of laser light illumination. In this extensive review, we focused on the methods and results of ALA-PDT for treating malignant gliomas in experimental conditions. The biological mechanisms, the effects on tumour and normal brain tissue, and finally the critical issues to optimize the efficacy of ALA-PDT were discussed.
\end{abstract}




\section{Introduction:}

Glioblastoma (GBM) is the most common and deadliest malignant brain tumour. The current standard of treatment for GBM involves maximal surgical resection followed by radiation therapy (RT) and concomitant chemotherapy with oral cytotoxic chemotherapy, temozolamide (TMZ), followed by maintenance administration of TMZ. The median survival for primary GBM with this combined treatment is 15 months (versus 12 months with RT only) and the 2-year survival rate increases to $26 \%$ compared to $10 \%$ in RT arm $[1,2]$. The three main causes of the poor prognosis associated with GBM are: (1) the tumour cells extensively infiltrate the surrounding brain tissue, limiting the efficacy of surgical resection; (2) the blood-brain barrier (BBB) prevents the optimal delivery of chemotherapy agents; (3) GBM is refractory to most cancer cytotoxic agents or rapidly develops resistance.

In the event of recurrences, associated with a poorer prognosis, other treatments added to an optional new surgery may be proposed. They include carmustine implants, TMZ rechallenges, or others chemotherapy such as bevacizumab [3-5]. Novel strategies, such as fluorescence-guided resection (FGR) [6], immunotherapy [7], gene therapy [8], anti-angiogeneic agents or targeting of growth-promoting pathways [9], provide additional avenues to increase survival benefits.

During the last decade, photodynamic therapy (PDT) has emerged as a promising treatment strategy in the management of various cancers in urology, gynecology or dermatology[10-13]. PDT relies on the selective accumulation of photosensitizers (PS) in tumour to generate the destruction of the cells. Illumination of the tumour with an excitation light tuned to the absorption band of the PS leads to the tumour destruction while sparing normal tissues. Thus the efficacy of PDT depends on numerous parameters, including tissue oxygenation status, photosensitizer concentration, and light regimen.

The mechanisms of PDT-mediated tumour destruction involve a direct cellular toxic effect, vascular damages and an immune reaction, which might be important for long-term tumour control $[14,15]$. In light of our work on developing interstitial PDT in a U87 glioma rodent model, we present an extensive review of the experimental use of PDT in high grade gliomas, with a specific focus on the use of 5-aminolevulinic acid induced Protoporphyrin IX, as well as a detailed study of the illumination and oxygenation conditions.

\section{PDT of gliomas}

\section{a. The choice of photosensitizer}

Ideal PS should absorb light in the red or far-red wavelengths, for increased tissue penetration, and should have the ability to cross the BBB. In addition, an ideal PS candidate would offer high tumour tissue selectivity, reduced skin and systemic toxicity through a rapid elimination of the PS by the patient $[14,16]$. Many compounds have already been investigated for PDT of gliomas.

Hematoporphyrin derivative (HpD; Photofrin () ) was the first PS to be studied reported in detail [16, 17]. Despite its interesting characteristics, poor tumour selectivity and long-lasting skin photosensitivity constituted several drawbacks that led to the development of new PS [18, 19]. Compounds such as m-THPC (m-tetrahydroxuphenyl chlorin, Foscan $\AA$ ), 5-ALA (5-aminolevulinic acid), BOPP (boronated porphyrin) and talaporfin sodium have been mainly used in clinical trials for 
gliomas (Table 1)[6, 20-26]. Their activation peaks are respectively at 652, 635 and $630 \mathrm{~nm}$. Others PS have been assessed in experimental studies, including hypericin, SIM01 and Pc4 [27, 28].

5-ALA is a second-generation PS that is already authorized in European Union for fluorescenceguided resection[20, 29]. It is a precursor that is converted into the potent photosensitizer, protoporphyrin IX (PpIX), as a part of the endogenous heme pathway. Under physiological conditions, the next stage in the cycle is the insertion of iron to convert PpIX into heme, which controls endogenous production of 5-ALA via a feedback inhibition [30]. Several mechanisms, including low expression of ferrochelatase in malignant tumours are correlated with higher concentration of PpIX in the tumour tissue than in the healthy brain [31-34]. Thus, an exogenous administration of 5-ALA would circumvent the feedback inhibition leading to an intracellular accumulation of PpIX [31]. In experimental studies, the average ratio of tumour-to-normal tissue PpIX concentration, estimated from the PpIX fluorescence, varied from 6:1 to 10:1. Angell-Peterson et al. reported a higher ratio of 200:1 in BD-IX rats bearing BT4C tumours after 5-ALA intraperitoneal injection [35-39].

Histological examination of human brain tissue samples acquired during ALA-FGR of high-grade gliomas exhibited a good correlation between the increased fluorescence and the cell density[40]. Moreover, the PpIX had a superior localization in high-grade glioma than in low-grade glioma or normal brain. These results indicated that the PpIX fluorescence was a sensitive tool for the detection of malignant gliomas [40,41]. The sensitivity of 5-ALA fluorescence for high-grade gliomas ranged from 57 to $85 \%$, correlated with the proportion of GBM [6, 42, 43]. False positive tissues were rare and associated with important inflammatory infiltration [41].

Another benefit of using 5-ALA for PDT is that PpIX was rapidly eliminated from the body, limiting the risk of skin photosensitization to 48 hours [44]. Elimination of the PS, monitored through photobleaching, was total: at the end of illumination, the PpIX was photodegraded to below 95\% of its initial level [45]. In contrast, others clinically used PS, mainly Phototofrin $₫$ or Foscan $\AA$, have left the patient at risk of toxic reactions following sunlight exposure for several weeks. Moreover, adverse effects considered to be drug-related to 5-ALA, were rarely reported: they included transient abnormal liver functions, nausea, and anecdotally generalized oedema requiring monitoring in an intensive care unit $[6,44-47]$. In summary, due to its high tumour selectivity, short half-life and lowlevel side effects, 5-ALA presented many of the characteristics we have identified for an ideal PS and should be intensively explored in the context of brain PDT for high-grade gliomas.

\section{b. Experimental models used in PDT of gliomas}

To date the experimental studies of PDT of glioblastoma have mainly been carried out in vitro on cell cultures or on multicell tumour spheroids. Spheroids were relevant in vitro models of glioma due to their three-dimensional spatial variations in oxygenation, nutritional, metabolic and proliferation status [37, 48-51]. They were able to mimic many characteristics of solid tumours and the observed trends could be reproduced in animal models. Moreover, glioma cells may be used in vivo after their stereotactic graft into animals brain.

The histological features of some experimental animal models of glioma, including syngeneic and xenogeneic models, were analyzed (Table 2) [52-54]. U87and U251 human GBM cells and GL26, CNS1, C6 murine cells were the most commonly reported. U87 human tumours exhibited profuse angiogenesis and a panel of marker proteins [55], allowing therapeutic test approaches that target neovascularization and immune reaction. Thus, xenotransplantation of U87 cells into rat brain 
provided a simple and well-defined rat model of fast-growing glioma for experimental studies of human malignant brain tumours.

Due to the lack of glial tumour for rabbits, VX2 carcinoma was chosen for its characteristics relevant to human GBM [56, 57]. Dogs bearing spontaneous GBM, such as Boston terriers and Boxers, could be an attractive large animal model but the possibility of recruiting GBM bearing dogs remains challenging [52].

In summary, the experimental models most commonly used in PDT of gliomas are: C6/CNS1/BT4C murine xenografts, human cells U87/U251/ACBT xenografts, 9L gliosarcoma and VX2 rabbit tumours.

\section{Review of experimental results}

\section{a. Photochemistry:}

Once a PS is illuminated with an exciting light source tuned at the absorption wavelength of interest, the ground state of the PS is excited to a singlet state of higher energy. Most PS has these two modes of relaxation: 1) emission of a fluorescent photon and 2) formation of a photochemically active molecule $[14,16]$.

On the first hand, the PS returns to its ground state by emitting a fluorescent photon. On the other hand, the relaxation mode from excited to ground state consists of an intermediate system with a sequence of photochemical responses, known as type I or type II reactions. These reactions generate highly reactive oxygen species from the PS, particularly a singlet oxygen $\left({ }^{1} \mathrm{O}_{2}\right)$, which in turn leads to cell death. The reactive molecules released in the cells cause mitochondrial and nuclear DNA damages. The analysis with chromatography or spectrophotometer of porphyrin after 5-ALA administration showed a high majority of PpIX $[38,58]$. The study of PpIX kinetic was facilitated by the use of its fluorescence properties.

\section{b. 5-ALA pharmacokinetics and biodistribution:}

Vanderveen et al. showed a correlation between the fluorescence intensity of PpIX and the dose of administered 5-ALA [59]: the fluorescence kinetics after oral ALA administration was similar to that after intravenous administration, although a higher dose was necessary to achieve the same tissue concentration of PpIX [60]. The concentration in brain tumour was higher than in normal tissue, and adequate for an efficient PDT [40, 61].

The time course distribution of fluorescence intensity in the rat brain tumour and vasogenic oedema was investigated and showed a peak in intensity between 3 to 6 hours after systemic 5-ALA administration. In the same manner, the optimal timing of fluorescence imaging of 5-ALA-PDT of brain tumours was determined to be within the period of 3-6 h, for best efficacy and selectivity [39, $61,62]$. Beyond the period of $6 \mathrm{~h}$, maximum fluorescence was detected at the area of the oedema surrounding the tumour. The metabolism of 5-ALA to porphyrins in normal brain tissue was negligible but notable fluorescence could be detected within white-matter tracks. The factors involved in the distribution of PS in the oedema may be the transport of porphyrins made in the tumour by bulk flow $[18,38,39,61,62]$ and the high sensitivity of porphyrins to the connective tissue components $[63,64]$. But the risk of diffusion of PpIX would be limited to $1.5 \mathrm{~mm}$ around tumour in the brain. Such a diffusion length is acceptable in clinical therapy since this area corresponds to an infiltrating tissue $[39,61]$. 


\section{c. ALA distribution in the tumour:}

The evolution of fluorescence after 5-ALA administration indicated that the production of PpIX could be heterogeneous in the tumour $[38,39,65]$. Images of PpIX fluorescence in spheroids displayed a heterogeneous distribution, with higher intensity in outer rim, according to their three-dimensional variations [48]. In other studies, the fluorescence was often found to be homogeneous in tumour bearing rats. However, a number of studies reported different patterns [38, 39, 65]. An increased production of PpIX was visible in the tumour margins in the 9L tumours whereas the fluorescence was confined to the bulk tumour tissue in C6 and U251 tumours. The presence of PpIX in the tumour margin could be linked to the extensive perivascular infiltration, which characteristics the 9L tumours [38]. The fluorescence in C6 central region was often patchy with non-fluorescent areas due to necrosis. Note that in case of fluoro-guided surgery, this observation may be of minor relevance because necrosis can easily be distinguished [39]. However interstitial PDT in these conditions could lead to an incomplete destruction of the tumour with early recurrences.

Systematically administered 5-ALA did not accumulate in sufficient quantities in infiltrating cells of a normal brain that is protected by an intact BBB [66]. These isolated cells posed a greater concern as they account for tumour recurrence [5]. Nonetheless, PDT could induce a significant immune reaction and be associated with a new therapy, which might kill isolated tumour cells that initially survived to PDT $[45,67-70]$.

\section{d. Effects of PDT}

\section{i. Effects of PDT on Glioma:}

Selective damages of experimental gliomas as a result of ALA-PDT have been reported [61]. Photodynamic damages were observed at the site of the tumour, which was previously determined on MR imaging, without significant lesion in the normal brain [61]. Thus, ALA-PDT was found to reduce the tumour residual volume after surgery, delay the recurrence and increase survival of tumour bearing rabbits [71]. However no direct effect of PDT could be found in tumour cells infiltrating normal brain with an intact BBB [66]. Moreover in some cases, residual foci of tumour cells were contained within the apparently damaged tumour tissue, which supported the results of the studies of the PpIX fluorescence distribution [37, 38, 61, 72].

The three main mechanisms of PDT-mediated tumour destruction included 1) direct cellular toxic effect, 2) vascular damage and 3) immune reaction.

The direct PDT effects seemed to depend on illumination regimen: acute ALA-PDT preferentially may induce necrosis whereas extended PDT may enhance induction of apoptosis $[36,57,73,74]$. The cytotoxic damages instigated the inflammatory and immune responses participating in the tissue destructive action. Thus they induced the development of an adaptive immune response associated with efficient recognition of tumour antigens. Some studies had also shown the existence of immune memory cells corresponding to long lasting systemic immunity $[75,76]$.

Vascular effects varied with different PS. For instance, HpD mediated PDT caused vessels constriction and thrombus formation by platelets activation and release of thromboxane [77]. These effects included components related to damage of the vascular endothelium. In contrast, ALA-PDT may lead to vessel constriction by a decrease of nitric oxygen concentration without endothelial 
lesion [78]. Microvascular collapse could lead to persistent post-PDT hypoxia and could contribute to long-term tumour control [79].

Moreover, photo-oxidative lesions generated by PDT resulted in a rapid cell death that alerts host's immune system [15, 70]. Hence, tumour PDT induced a strong host response mediated by innate immune system and characterized by inflammatory and acute phase responses that led to the development of adaptive immune response recognizing the treated tumour as its target [70].

Finally, ALA-PDT could affect the invasiveness of GBM. In vitro, this mechanism seemed to be independent of direct phototoxicity because the viability of cells remained high. The changes induced in cytoskeleton and in the expression of molecules involved in matrix invasion might cause suppression of migratory and invasive capacities of tumour cells in spheroids [49, 80]. These mechanisms may be affected by fluence rate for a given total light dose. These appeared to be a long lasting effect but their fundamental mechanisms were not well understood $[49,80]$.

\section{ii. Effects of ALA-PDT on normal brain:}

In the experimental conditions considered, no significant damage to normal brain was induced in response to $200 \mathrm{~J} / \mathrm{cm}^{2}$, the highest dose of ALA-PDT used in clinical settings [61]. The same results were demonstrated in a model of vasogenic oedema with the same PS. In contrast, Photofrin ${ }^{\circledR}$ mediated PDT in the same model potentially caused extensive damages in the normal brain with neurological deficits [23]. With higher power, some histological changes such as hemorrhages, intravascular thrombosis, and death of neurons appeared in normal brain $[36,61,81]$.

Finally, the risk of light induced thermal effects was investigated. This thermal effect seemed to potentiate the efficacy of PDT $[82,83]$. Several studies noted that temperatures above $44^{\circ} \mathrm{C}$ were not tolerated by the brain [84]. However maximal temperature elevation of $42-43^{\circ} \mathrm{C}$ could be reached in rats model, without any damage observed $[21,85]$.

\section{iii. ALA-PDT induce oedema:}

5-ALA-PDT generated cerebral oedema, which could be of two types. In tumour and brain around tumour, it seemed to be cytotoxic oedema with poor response to steroids. Vasogenic oedema might progress in ipsilateral normal brain after illumination [86, 87]. The use of steroids resulted in a dramatic decrease in this oedema. Consequently, PDT should be restricted to small tumours or after surgical cytoreduction. In case of no-surgical tumour with significant mass effect, PDT may lead to decompensation of an increased intracranial pressure [86-88].

\section{e. Illumination schemes:}

Several illumination designs had been investigated to improve the efficacy of PDT. Indeed, high fluence rates, such as $26 \mathrm{~J}$ of radiant energy at $30 \mathrm{~mW}$, in rat models, resulted in rapid depletion of tumour oxygen, reduced the primary cytotoxic process of PDT and affected tumour control $[89,90]$. Potential solutions to this problem included the use of other light delivery schemes, such as low fluence rate PDT (for example 26 J at $4.8 \mathrm{~mW}$ in rat models), metronomic PDT [57], repetitive PDT or fractioned PDT.

In vitro, low fluence rate (FR) protocols could induce an increased tumour growth inhibition (from $20 \%$ with high FR to $90 \%$ [50]. Low-FR ALA-PDT, preserving the oxygen level, may improve the 
survival and the volume of necrosis volume of tumour-bearing animals [37]. Moreover, this regimen resulted in the generation of inflammatory and apoptotic response in tumour bearing animals[37, 91].

The combined delivery of both PS and light at low FR during an extended period, also referred to as metronomic PDT (mPDT), was also reported [92]. The aim was to increase selective apoptosis to kill tumour cells. This required prolonged illumination with implantation of an optic fiber for several days. The light was applied continuously either with an ultra low FR or in repetitive illuminations. In the case of ALA-PDT, additional administrations of PS were required because of the photobleaching mechanism and rapid elimination of the PS by the patient. Only a limited number of studies were available, but the first experiments in tumour-bearing rabbits appeared promising and technically feasible $[56,57,93]$.

Repetitive ALA-PDT separated by several weeks also proved its efficacy in spheroids and rat models $[94,95]$. The in vitro response to repetitive treatment at relatively long intervals (weeks) was better than either daily fractioned PDT or single-treatment regimens [95, 96]. Thus a single PDT treatment may not be sufficient to prevent recurrence, because of the short doubling time of malignant gliomas. However a repetitive illumination required to leave a fiber-based device during several weeks, or to insert a new tip at each illumination. In contrast, light fractionation with a short-term interval could be easily achieved and produced beneficial results. In animal models of breast and ovarian cancer, the schemes with one or more interruptions of few seconds or minutes were promising $[50,59,97,98]$. They may reverse vasoconstriction induced by PDT and allow reoxygenation during the dark period, leading to more reactive oxygen species formation. Fractioned illumination seemed to be more effective than continuous light to increase necrosis, but the results remained controversial [99-103]: in the normal rat colon, the area of PDT necrosis could be increased by a factor of three with a fractioned illumination [99], but some studies using Photofrin® or m-THPC showed no benefit in gliomas $[102,104]$. Therefore further investigations about 5-ALAPDT will be required in GBM models, since this effect seemed to be dependent on the PS used [59, 99, 105].

\section{f. Experimental data on oxygen}

Oxygenation status seemed to be the main factor limiting PDT efficacy related to the formation of reactive oxygen species [106]. Malignant gliomas are indeed notable for areas of necrosis and hypoxia, due to a highly abnormal vasculature [107]. In addition, vasoconstriction induced by PDT treatment increased local hypoxia, which interfered with the effects of PDT. In vitro, the oxygenation status of spheroids strongly determined the efficacy of PDT [51]. In some studies, animals were subjected to hyperbaric oxygen during Photofrin $®$-PDT for breast tumour. Hyperoxygenation could then improve PDT efficacy by oxygenating both preexisting and treatment-induced hypoxic cells [108-110]. Modulation of light delivery as low FR, metronomic PDT or fractioned PDT constituted an attempt to address this issue but the management of such protocols could be difficult in clinical settings.

\section{g. Other ways of research}

\section{iv. The Photosensitizer:}

Some strategies had been developed to facilitate the transport of ALA across the BBB in order to increase the efficiency of ALA-PDT. Various ALA esters with higher lipophilicity had been 
investigated as PS in pre-clinical models [48, 111, 112]. It had been shown that ALA hexylester reduced the dose required for a given level of PpIX production $[111,112]$. However, as a result of the possible poor specificity of ALA-esters for brain tumour tissue, an accumulation of PpIX in white matter could be observed [113]. The more lipophilic ALA-esters that penetrated through the BBB better than the polar ALA had a higher affinity for the lipid-rich white matter. Due to a non-specific accumulation of this PS in white matter, this approach appeared unsuitable for the treatment of gliomas.

Thus, to improve transport across the BBB, the treatment was performed with direct intratumoural injection of 5-ALA [66]. However, this mode of drug delivery was associated with high toxicity, high mortality and poor efficacy, as a result of rapid ALA diffusion in the brain parenchyma. In contrast, the delivery vehicles such as liposomes or nanoparticles showed promising results but remain to be investigated with 5-ALA [114].

Others studies investigated various strategies to improve the accumulation of PpIX in tumour cells. The combinations of ALA-PDT with iron chelator to prevent the formation of heme and the inhibition of ferrochelatase have shown interesting results by increasing the concentration of PpIX in tumour $[108,115,116]$. In contrast, both steroids and Phenytoin, common therapeutic molecules used in the management of GBM, could reduce ALA uptake and PpIX production $[117,118]$.

Finally, the combination of two different PS has been proposed to improve the global effect of PDT. The association of ALA and Photofrin ${ }^{\circledR}$ was investigated for the possible combination of their specific targets $[22,119]$. Endogenous PpIX is mainly formed within the mitochondria of tumour cells. Initial damage after illumination occurs in these cellular components [120]. In contrast, Photofrin $®$ was known to distribute mainly in the vascular stroma of the tumour. It led to the destruction of the vascular system during light exposure [121-123]. Therefore, tumour eradication would be more efficient with both interstitial and vascular targets [119].

\section{v. Vascular and immune effects:}

The vascular and immune PDT effects have also been investigated, in particular with Photofrin $($ PDT. PDT-induced cell hypoxia could limit the efficacy of this treatment as a result of vascular damage and photochemical oxygen consumption. Hypoxia may result in angiogenesis within rat brains, and subsequently, contribute to tumour regrowth [124, 125]. Several reports demonstrated that therapeutic doses of PDT could induce expression of HIF-1a and vascular endothelial growth factor (VEGF) and endothelial cell proliferation $[125,126]$. Thus, the combination of PDT with antiangiogeneic molecules may suppress angiogenesis, reduce tumour volume and prolong survival time of glioma xenograft animals $[124,126,127]$. These results were promising but other investigations are required to pinpoint these effects, especially with ALA-PDT.

PDT induces others tumour promoting factors as nuclear factor-kappaB (NF-KB), a transcription factor implicated in the regulation of many cellular processes and in the activation of anti-apoptotic factors in GBM. PDT combined with NF-KB inhibition may improve glioma cell death in animals [128]. On the other hand, PDT stimulated the immune response by inducing heat-shock proteins, and cyto- and chemokines [70, 129]. These elements led to the development of adaptive immune response based on the recognition of antigens of PDT-treated tumours. Thus, PDT could be exploited for the generation of additional tumour vaccines. Such treatments had been developed in vitro from PDT treated tumour cells and several studies had confirmed the efficacy of these vaccines generated by Hematoporphyrin-PDT in animal models [69, 130,131]. Further improvements are needed to 
maximize the protocols for the generation of these vaccines before their use in personalized medicine.

\section{Discussion}

\section{a. U87 human GBM xenografts model}

The U87 tumours failed to exhibit two hallmarks of the spontaneous GBM in human. Only small foci of necrosis were occasionally detected and their invasion pattern was not as diffuse as those in human GBM. Nevertheless, the U87 human tumours were characterized by profuse angiogenesis, which is the third histological feature of GBM [52]. Only few studies focused on the effects of ALAPDT on the U87 human GBM xenograft models, but they proved to be useful when assessing GBM neovascularization. Previous studies showed that low dose PDT could induce the proliferation of endothelial cells and the expression of VEGF in the brain adjacent to the tumour. Importantly, the efficacy of PDT may be diminished by its pro-angiogenic effects, which subsequently contribute to tumour regrowth $[124,126]$. Therefore, the U87 tumours could be useful in the evaluation of new anti-angiogenic therapeutic approaches or fractioned illumination that involved vascular mechanisms.

To our knowledge, no study has reported the uptake and distribution of the PpIX in the U87 human GBM xenograft models. Hence, it seems to be a prerequisite before the use of this model for PDT.

\section{b. Advantages of ALA induced PpIX.}

5-ALA has been authorized in the European Union for the FGR of malignant glioma since 2007. In contrast to Hpd or m-THPC, the side effects of ALA were mild at the recommended dosage [18, 19, 44]. ALA induced PPIX are natural compounds that are rapidly cleared from normal tissues, limiting skin photosensitivity to 1 or 2 days. Moreover, some studies demonstrated that ALA displayed a good tumour-selective uptake with high tumour to normal brain ratios while PpIX was not detectable in the normal brain after 5-ALA administration in healthy rats [35-39]. However, in some cases, porphyrins had been found into white matter fiber tracts bordering the tumour of GBM xenograft model $[38,39,45,61,72]$. This could be advantageous for applications in frontal tumour with an extension in the corpus callosum, in which PpIX could follow the white matters tracts and the PDT would have a higher efficacy than surgery alone. But in most cases, the PpIX spreading from the tumour was limited and the effects on normal brain were negligible.

In the same way, ALA-mediated PDT induced a lower oedema reaction than HpD-PDT for a given light dose. But some authors reported a significant cerebral oedema in response to ALA-PDT, even though morphological damages were restricted to tumour tissue $[86,87,132]$. This oedema could be dangerous after an interstitial PDT as it may result in increased intracranial pressure and respond only partially to steroid treatments. Furthers experimentations are still required to explain this phenomenon, especially as the light regimen could affect the development of oedema by following the pattern of cell death. In contrast to necrosis, apoptosis is characterized by the lack of inflammatory reactions and associated oedema. The rate of PDT-induced apoptosis may depend on the PS used and the illumination regimens $[37,73]$. The modulation of light dose in association with a steroid treatment could be very useful to prevent oedema[21].

In an effort to improve the efficacy of PDT, the combination of two PS had also been proposed. The association of two PS seemed to hold several advantages. First, this approach combined both 
vascular and interstitial targets of PS. Moreover, ALA-mediated PDT was highly effective in opening the BBB in a limited region surrounding the tumour, which containing infiltrating tumour cells [132]. It may also allow an additional PS to reach these cells and increase the efficacy of PDT $[86,87,132]$. Finally, these associations had been used in FGR followed by PDT of the surgical cavity during a few days. Eljamel et al. used ALA-FGR to reduce the gross tumour, immediately followed by repetitive illumination while the Photofrin $(\AA$ is still active in the remaining tumour cells [22]. Prolonged illumination could be limited with 5-ALA alone because of its short lifetime. Moreover, iterative administrations of 5-ALA could result in systemic toxicity [44]. Rather than such a prolonged treatment, fractioned illumination could easily be implemented in ALA-PDT of GBM. It consists in alternating light delivery into periods of "on" and "off" to allow tissues reoxygenation. It had shown an efficacy in non-Central Nervous System (CNS) Cancer [97-99]. This scheme had the potential to increase the amount of necrosis produced by PDT by a factor of three by short interruptions of the illumination $[99,101]$. This improvement afford by fractioned light delivery seemed to depend on the PS used. Some studies had demonstrated the greatest improvement with ALA in non-CNS cancer $[59,99,105]$. No benefit with $\mathrm{m}-$ THPC or Photofrin $®$ sensitization was found in GBM $[102,104]$. But, to our knowledge, no study had reported the effect of ALA-mediated PDT with fractioned illumination on GBM. Therefore further experimentations would be valuable.

In addition to cytotoxic effects, other mechanisms during ALA-mediated PDT may be involved in long-lasting stabilization.

First, ALA-mediated PDT may interfere with the invasiveness of GBM, affecting the migratory and invasive activity of glioma cells. This effect is mediated by changes induced in the cytoskeleton and the expression of molecules involved in matrix invasion. It could also delay recurrences $[49,80]$. However, a study reported an opposite effect of Photofrin@-mediated PDT into sensitized normal brain in a mouse model. Sub-lethal dose of Photofrin $®$-mediated PDT may facilitate U87 tumour growth and invasiveness through the microenvironment changes [133]. Due to these conflicting reports, further investigations are needed to determine the potential increased tumour cell migration following PDT.

Secondly, ALA-mediated PDT could increase tumour cells destruction via the activation of an immune response. Some studies suggested that it might induce the expression of cytokines and heat shock proteins (HSP) in response to oxidative stress $[69,70,129]$. The immune responses can act upon the cells that have not been killed by direct phototoxic effects, or be targeted by tumour vaccine or immunotherapy [69].

\section{c. Optimization of PS and light delivery}

Given the high inter-patient variations, it was necessary to enhance the individual dosimetry technique to avoid under-treatment. It should measure the real energy absorbed by the target tissue and the surrounding tissue, in association with the dose of PS and oxygen status. This concept required considering the heterogeneous optical properties, PS uptake, and light distribution [46, 134]. Some approaches presented a significant potential but optimizations were required to obtain a suitable procedure. A different approach could consist in using a device to measure $\mathrm{PO}_{2}$ in tumours, to evaluate whether sufficient light has been delivered [100, 135]. Indeed, it seemed that a documented decrease in $\mathrm{PO}_{2}$ at the time of treatment might be a valuable predictive value of necrosis. Khan et al have coined this technique "oximetry for chemotherapies" [100, 135]. However, treatment monitoring remains in its infancy. Moreover, the development of software and devices for the treatment-planning and stereotactic implantation procedures will be needed. In the same way 
that implantable balloon applicators were used for the tumour cavity illumination [136], implantable cannula or screw could secure the optical fiber path during iPDT.

Although ALA was considered safe, ALA-mediated PDT may have serious side effects such as significant hyperthermia. Even if unexpected activation of ALA outside the tumour tissue apparently never occurred $[39,61,62,85]$, the development of oedema induced a risk of increased intracranial pressure. This oedema seemed to depend on the PS used and the light regimen $[86,87,132]$.

After all, the delayed effects of these treatments were not completely elucidated and some authors suggested reverse effects such as the stimulation of tumour regrowth and invasiveness $[49,80]$.

\section{Conclusion}

We presented here an extensive review of experimental studies of ALA-PDT in Glioblastoma. Additionally to standard treatments for GBM, PDT holds great promise toward an improved local tumour control. ALA is already authorized in European Union for fluorescence-guided resection. Although ALA-mediated PDT seems to be encouraging, the procedure still requires optimizations.

References:

1. Stupp, R., et al., Radiotherapy plus concomitant and adjuvant temozolomide for glioblastoma. $\mathrm{N}$ Engl J Med, 2005. 352(10): p. 987-96.

2. Walker, M.D., et al., Randomized comparisons of radiotherapy and nitrosoureas for the treatment of malignant glioma after surgery. N Engl J Med, 1980. 303(23): p. 1323-9.

3. Clarke, J.L., et al., Randomized phase II trial of chemoradiotherapy followed by either dose-dense or metronomic temozolomide for newly diagnosed glioblastoma. J Clin Oncol, 2009. 27(23): p. 3861-7.

4. Anton, K., J.M. Baehring, and T. Mayer, Glioblastoma multiforme: overview of current treatment and future perspectives. Hematol Oncol Clin North Am, 2012. 26(4): p. 825-53.

5. Walker, M.D., et al., Evaluation of BCNU and/or radiotherapy in the treatment of anaplastic gliomas. A cooperative clinical trial. J Neurosurg, 1978. 49(3): p. 333-43.

6. Stummer, W., et al., Fluorescence-guided surgery with 5-aminolevulinic acid for resection of malignant glioma: a randomised controlled multicentre phase III trial. Lancet Oncol, 2006. 7(5): p. 392-401.

7. Chang, C.N., et al., A phase I/II clinical trial investigating the adverse and therapeutic effects of a postoperative autologous dendritic cell tumor vaccine in patients with malignant glioma. J Clin Neurosci, 2011. 18(8): p. 1048-54.

8. Rainov, N.G., A phase III clinical evaluation of herpes simplex virus type 1 thymidine kinase and ganciclovir gene therapy as an adjuvant to surgical resection and radiation in adults with previously untreated glioblastoma multiforme. Hum Gene Ther, 2000. 11(17): p. 2389-401.

9. Akhavan, D., T.F. Cloughesy, and P.S. Mischel, mTOR signaling in glioblastoma: lessons learned from bench to bedside. Neuro Oncol, 2010. 12(8): p. 882-9.

10. De Vijlder, H.C., et al., Light fractionation significantly improves the response of superficial basal cell carcinoma to aminolaevulinic acid photodynamic therapy: five-year follow-up of a randomized, prospective trial. Acta Derm Venereol, 2012. 92(6): p. 641-7.

11. Choi, M.C., et al., Fertility preservation via photodynamic therapy in young patients with earlystage uterine endometrial cancer: a long-term follow-up study. Int J Gynecol Cancer, 2013. 23(4): p. 698-704. 
12. Fukuhara, H., et al., The inhibition of ferrochelatase enhances 5-aminolevulinic acid-based photodynamic action for prostate cancer. Photodiagnosis Photodyn Ther, 2013. 10(4): p. 399409.

13. Svanberg, K., et al., Photodynamic therapy: superficial and interstitial illumination. J Biomed Opt, 2010. 15(4): p. 041502.

14. Stylli, S.S. and A.H. Kaye, Photodynamic therapy of cerebral glioma--a review Part I--a biological basis. J Clin Neurosci, 2006. 13(6): p. 615-25.

15. Castano, A.P., Mechanisms in photodynamic therapy: Part three-Photosensitizer pharmacokinetics, biodistribution, tumor localization and modes of tumor destruction. Photodiagnosis and Photodynamic Therapy, 2005(2): p. 91-106.

16. Castano, A.P., et al, Mechanisms in photodynamic therapy: Part one- Photosensitizers, photochemistry and cellular localization. Photodiagnosis and Photodynamic Therapy, 2004(1): p. 279-93.

17. Stylli, S.S., et al., Photodynamic therapy of high grade glioma - long term survival. J Clin Neurosci, 2005. 12(4): p. 389-98.

18. Stummer, W., et al., Kinetics of Photofrin II in perifocal brain edema. Neurosurgery, 1993. 33(6): p. 1075-81; discussion 1081-2.

19. Hebeda, K.M., et al., Fluorescence localization in tumor and normal brain after intratumoral injection of haematoporphyrin derivative into rat brain tumor. J Photochem Photobiol B, 1995. 27(1): p. 85-92.

20. Kostron, H., Photodynamic diagnosis and therapy and the brain. Methods Mol Biol, 2010. 635: p. 261-80.

21. Beck, T.J., et al., Interstitial photodynamic therapy of nonresectable malignant glioma recurrences using 5-aminolevulinic acid induced protoporphyrin IX. Lasers Surg Med, 2007. 39(5): p. 386-93.

22. Eljamel, M.S., C. Goodman, and H. Moseley, ALA and Photofrin fluorescence-guided resection and repetitive PDT in glioblastoma multiforme: a single centre Phase III randomised controlled trial. Lasers Med Sci, 2008. 23(4): p. 361-7.

23. Rosenthal, M.A., et al., Phase I and pharmacokinetic study of photodynamic therapy for highgrade gliomas using a novel boronated porphyrin. J Clin Oncol, 2001. 19(2): p. 519-24.

24. Hill, J.S., et al., Selective tumor kill of cerebral glioma by photodynamic therapy using a boronated porphyrin photosensitizer. Proc Natl Acad Sci U S A, 1995. 92(26): p. 12126-30.

25. Hill, J.S., et al., Selective tumor uptake of a boronated porphyrin in an animal model of cerebral glioma. Proc Natl Acad Sci U S A, 1992. 89(5): p. 1785-9.

26. Muragaki, Y., et al., Phase II clinical study on intraoperative photodynamic therapy with talaporfin sodium and semiconductor laser in patients with malignant brain tumors. J Neurosurg, 2013. 119(4): p. 845-52.

27. Huntosova, V., et al., Cell death response of U87 glioma cells on hypericin photoactivation is mediated by dynamics of hypericin subcellular distribution and its aggregation in cellular organelles. Photochem Photobiol Sci, 2012. 11(9): p. 1428-36.

28. Castano, A.P., Mechanisms in photodynamic therapy: part two-cellular signaling, cell metabolism and modes of cell death. Photodiagnosis and Photodynamic Therapy, 2005(2): p. 1-23.

29. Eljamel, S., Photodynamic applications in brain tumors: a comprehensive review of the literature. Photodiagnosis Photodyn Ther, 2010. 7(2): p. 76-85.

30. Rimington, C., Porphyrin and haem biosynthesis and its control. Acta Med Scand Suppl, 1966. 445: p. 11-24.

31. Peng, Q., et al., 5-Aminolevulinic acid-based photodynamic therapy. Clinical research and future challenges. Cancer, 1997. 79(12): p. 2282-308. 
32. Collaud, S., et al., On the selectivity of 5-aminolevulinic acid-induced protoporphyrin IX formation. Curr Med Chem Anticancer Agents, 2004. 4(3): p. 301-16.

33. Greenbaum, L., et al., Nuclear distribution of porphobilinogen deaminase (PBGD) in glioma cells: a regulatory role in cancer transformation? Br J Cancer, 2002. 86(6): p. 1006-11.

34. Ennis, S.R., et al., Transport of 5-aminolevulinic acid between blood and brain. Brain Res, 2003. 959(2): p. 226-34.

35. Obwegeser, A., R. Jakober, and H. Kostron, Uptake and kinetics of 14C-labelled metatetrahydroxyphenylchlorin and 5-aminolaevulinic acid in the C6 rat glioma model. Br J Cancer, 1998. 78(6): p. 733-8.

36. Lilge, L., et al., The sensitivity of normal brain and intracranially implanted VX2 tumor to interstitial photodynamic therapy. Br J Cancer, 1996. 73(3): p. 332-43.

37. Angell-Petersen, E., et al., Influence of light fluence rate on the effects of photodynamic therapy in an orthotopic rat glioma model. J Neurosurg, 2006. 104(1): p. 109-17.

38. Hebeda, K.M., et al., 5-Aminolevulinic acid induced endogenous porphyrin fluorescence in $9 \mathrm{~L}$ and C6 brain tumors and in the normal rat brain. Acta Neurochir (Wien), 1998. 140(5): p. 503-12; discussion 512-3.

39. Stummer, W., et al., In vitro and in vivo porphyrin accumulation by C6 glioma cells after exposure to 5-aminolevulinic acid. J Photochem Photobiol B, 1998. 45(2-3): p. 160-9.

40. Johansson, A., et al., 5-Aminolevulinic acid-induced protoporphyrin IX levels in tissue of human malignant brain tumors. Photochem Photobiol, 2010. 86(6): p. 1373-8.

41. Utsuki, S., et al., Histological examination of false positive tissue resection using 5-aminolevulinic acid-induced fluorescence guidance. Neurol Med Chir (Tokyo), 2007. 47(5): p. 210-3; discussion 213-4.

42. Floeth, F.W., et al., Comparison of (18)F-FET PET and 5-ALA fluorescence in cerebral gliomas. Eur J Nucl Med Mol Imaging, 2010. 38(4): p. 731-41.

43. Ewelt, C., et al., Finding the anaplastic focus in diffuse gliomas: the value of Gd-DTPA enhanced MRI, FET-PET, and intraoperative, ALA-derived tissue fluorescence. Clin Neurol Neurosurg, 2011. 113(7): p. 541-7.

44. Webber, J., D. Kessel, and D. Fromm, Side effects and photosensitization of human tissues after aminolevulinic acid. J Surg Res, 1997. 68(1): p. 31-7.

45. Stepp, H., et al., ALA and malignant glioma: fluorescence-guided resection and photodynamic treatment. J Environ Pathol Toxicol Oncol, 2007. 26(2): p. 157-64.

46. Hennig, G., H. Stepp, and A. Johansson, Photobleaching-based method to individualize irradiation time during interstitial 5-aminolevulinic acid photodynamic therapy. Photodiagnosis Photodyn Ther, 2011. 8(3): p. 275-81.

47. Hefti, M., et al., 5-aminolevulinic acid induced protoporphyrin IX fluorescence in high-grade glioma surgery: a one-year experience at a single institutuion. Swiss Med Wkly, 2008. 138(1112): p. 180-5.

48. Bigelow, C.E., et al., ALA- and ALA-hexylester-induced protoporphyrin IX fluorescence and distribution in multicell tumor spheroids. Br J Cancer, 2001. 85(5): p. 727-34.

49. Hirschberg, H., et al., Effects of ALA-mediated photodynamic therapy on the invasiveness of human glioma cells. Lasers Surg Med, 2006. 38(10): p. 939-45.

50. Mathews, M.S., et al., The effects of ultra low fluence rate single and repetitive photodynamic therapy on glioma spheroids. Lasers Surg Med, 2009. 41(8): p. 578-84.

51. Zelenkov, P., et al., Acute morphological sequelae of photodynamic therapy with 5aminolevulinic acid in the C6 spheroid model. J Neurooncol, 2007. 82(1): p. 49-60.

52. Candolfi, M., et al., Intracranial glioblastoma models in preclinical neuro-oncology: neuropathological characterization and tumor progression. J Neurooncol, 2007. 85(2): p. 13348. 
53. Sun, L., Animal Models of Glioma, Glioma - Exploring Its Biology and Practical Relevance, ed. D.A.G. (Ed.). 2011.

54. Barth, R.F. and B. Kaur, Rat brain tumor models in experimental neuro-oncology: the C6, 9L, T9, RG2, F98, BT4C, RT-2 and CNS-1 gliomas. J Neurooncol, 2009. 94(3): p. 299-312.

55. Strojnik, T., R. Kavalar, and T.T. Lah, Experimental model and immunohistochemical analyses of U87 human glioblastoma cell xenografts in immunosuppressed rat brains. Anticancer Res, 2006. 26(4B): p. 2887-900.

56. Bogaards, A., et al., Fluorescence image-guided brain tumor resection with adjuvant metronomic photodynamic therapy: pre-clinical model and technology development. Photochem Photobiol Sci, 2005. 4(5): p. 438-42.

57. Bisland, S.K., et al., Metronomic photodynamic therapy as a new paradigm for photodynamic therapy: rationale and preclinical evaluation of technical feasibility for treating malignant brain tumors. Photochem Photobiol, 2004. 80: p. 22-30.

58. Pottier, R.H., et al., Non-invasive technique for obtaining fluorescence excitation and emission spectra in vivo. Photochem Photobiol, 1986. 44(5): p. 679-87.

59. Van der Veen, N., H.L. van Leengoed, and W.M. Star, In vivo fluorescence kinetics and photodynamic therapy using 5-aminolaevulinic acid-induced porphyrin: increased damage after multiple irradiations. Br J Cancer, 1994. 70(5): p. 867-72.

60. Loh, C.S., et al., Oral versus intravenous administration of 5-aminolaevulinic acid for photodynamic therapy. Br J Cancer, 1993. 68(1): p. 41-51.

61. Olzowy, B., et al., Photoirradiation therapy of experimental malignant glioma with 5aminolevulinic acid. J Neurosurg, 2002. 97(4): p. 970-6.

62. Tsurubuchi, T., et al., The optimization of fluorescence imaging of brain tumor tissue differentiated from brain edema--in vivo kinetic study of 5-aminolevulinic acid and talaporfin sodium. Photodiagnosis Photodyn Ther, 2009. 6(1): p. 19-27.

63. El-Far, M.A. and N.R. Pimstone, The interaction of tumor-localizing porphyrins with collagen, elastin, gelatin, fibrin and fibrinogen. Cell Biochem Funct, 1985. 3(2): p. 115-9.

64. Musser, D.A., J.M. Wagner, and M. Datta-Gupta, The interaction of tumor localizing porphyrins with collagen and elastin. Res Commun Chem Pathol Pharmacol, 1982. 36(2): p. 251-9.

65. Gibbs-Strauss, S.L., et al., Noninvasive measurement of aminolevulinic acid-induced protoporphyrin IX fluorescence allowing detection of murine glioma in vivo. J Biomed Opt, 2009. 14(1): p. 014007.

66. Madsen, S.J., et al., Photodynamic therapy of newly implanted glioma cells in the rat brain. Lasers Surg Med, 2006. 38(5): p. 540-8.

67. Bellnier, D.A., Potentiation of photodynamic therapy in mice with recombinant human tumor necrosis factor-alpha. J Photochem Photobiol B, 1991. 8(2): p. 203-10.

68. Korbelik, M. and G. Krosl, Enhanced macrophage cytotoxicity against tumor cells treated with photodynamic therapy. Photochem Photobiol, 1994. 60(5): p. 497-502.

69. Korbelik, M., B. Stott, and J. Sun, Photodynamic therapy-generated vaccines: relevance of tumor cell death expression. Br J Cancer, 2007. 97(10): p. 1381-7.

70. Korbelik, M., PDT-associated host response and its role in the therapy outcome. Lasers Surg Med, 2006. 38(5): p. 500-8.

71. Xiao, $\mathrm{H}$. , et al., 5-Amino-4-oxopentanoic acid photodynamic diagnosis guided microsurgery and photodynamic therapy on VX2 brain tumor implanted in a rabbit model. Chin Med J (Engl), 2009. 122(11): p. 1316-21.

72. Hebeda, K.M., et al., Damage to tumor and brain by interstitial photodynamic therapy in the $9 \mathrm{~L}$ rat tumor model comparing intravenous and intratumoral administration of the photosensitiser. Acta Neurochir (Wien), 1998. 140(5): p. 495-501. 
73. Lilge, L., M. Portnoy, and B.C. Wilson, Apoptosis induced in vivo by photodynamic therapy in normal brain and intracranial tumor tissue. Br J Cancer, 2000. 83(8): p. 1110-7.

74. Coupienne, I., et al., 5-ALA-PDT induces RIP3-dependent necrosis in glioblastoma. Photochem Photobiol Sci, 2011. 10(12): p. 1868-78.

75. Korbelik, M. and I. Cecic, Contribution of myeloid and lymphoid host cells to the curative outcome of mouse sarcoma treatment by photodynamic therapy. Cancer Lett, 1999. 137(1): p. 91-8.

76. Korbelik, M. and G.J. Dougherty, Photodynamic therapy-mediated immune response against subcutaneous mouse tumors. Cancer Res, 1999. 59(8): p. 1941-6.

77. Fingar, V.H., et al., The effects of thromboxane inhibitors on the microvascular and tumor response to photodynamic therapy. Photochem Photobiol, 1993. 58(3): p. 393-9.

78. Gilissen, M.J., et al., Effect of photodynamic therapy on the endothelium-dependent relaxation of isolated rat aortas. Cancer Res, 1993. 53(11): p. 2548-52.

79. Henderson, B.W. and V.H. Fingar, Relationship of tumor hypoxia and response to photodynamic treatment in an experimental mouse tumor. Cancer Res, 1987. 47(12): p. 3110-4.

80. Etminan, N., et al., Modulation of migratory activity and invasiveness of human glioma spheroids following 5-aminolevulinic acid-based photodynamic treatment. Laboratory investigation. J Neurosurg, 2011. 115(2): p. 281-8.

81. Ji, Y., et al., Interstitial photoradiation injury of normal brain. Lasers Surg Med, 1992. 12(4): p. 425-31.

82. Mang, T.S. and T.J. Dougherty, Time and sequence dependent influence of in vitro photodynamic therapy (PDT) survival by hyperthermia. Photochem Photobiol, 1985. 42(5): p. 533-40.

83. Waldow, S.M., B.W. Henderson, and T.J. Dougherty, Potentiation of photodynamic therapy by heat: effect of sequence and time interval between treatments in vivo. Lasers Surg Med, 1985. 5(2): p. 83-94.

84. Salcman, M. and G.M. Samaras, Hyperthermia for brain tumors: biophysical rationale. Neurosurgery, 1981. 9(3): p. 327-35.

85. Elias, Z., et al., Hyperthermia from interstitial laser irradiation in normal rat brain. Lasers Surg Med, 1987. 7(4): p. 370-5.

86. Mathews, M.S., et al., Cerebral edema following photodynamic therapy using endogenous and exogenous photosensitizers in normal brain. Lasers Surg Med, 2011. 43(9): p. 892-900.

87. Ito, S., et al., Oedema formation in experimental photo-irradiation therapy of brain tumors using 5-ALA. Acta Neurochir (Wien), 2005. 147(1): p. 57-65; discussion 65.

88. Klatzo, I., A. Piraux, and E.J. Laskowski, The relationship between edema, blood-brain-barrier and tissue elements in a local brain injury. J Neuropathol Exp Neurol, 1958. 17(4): p. 548-64.

89. Gibson, S.L., et al., Effects of various photoradiation regimens on the antitumor efficacy of photodynamic therapy for R3230AC mammary carcinomas. Cancer Res, 1990. 50(22): p. 723641.

90. Sitnik, T.M., J.A. Hampton, and B.W. Henderson, Reduction of tumor oxygenation during and after photodynamic therapy in vivo: effects of fluence rate. Br J Cancer, 1998. 77(9): p. 1386-94.

91. Henderson, B.W., et al., Choice of oxygen-conserving treatment regimen determines the inflammatory response and outcome of photodynamic therapy of tumors. Cancer Res, 2004. 64(6): p. 2120-6.

92. Wilson, B., Metronomic photodynamic therapy (mPDT): concepts and technical feasibility in brain tumor,. proc SPIE, 2003. 2003(4952): p. 23-31.

93. Alqawi, O., Espiritu, M., Singh, G., Molecular mechanisms associated with ALA-PDT of brain tumor cells. Photodynamic therapy: back to the future, 2009. 7380.

94. Madsen, S.J., et al., Repetitive 5-aminolevulinic acid-mediated photodynamic therapy on human glioma spheroids. J Neurooncol, 2003. 62(3): p. 243-50. 
95. Hirschberg, H., et al., Repetitive photodynamic therapy of malignant brain tumors. J Environ Pathol Toxicol Oncol, 2006. 25(1-2): p. 261-79.

96. Hirschberg, H., et al., Enhanced cytotoxic effects of 5-aminolevulinic acid-mediated photodynamic therapy by concurrent hyperthermia in glioma spheroids. J Neurooncol, 2004. 70(3): p. 289-99.

97. Estevez, J.P., et al., Continuous or fractionated photodynamic therapy? Comparison of three PDT schemes for ovarian peritoneal micrometastasis treatment in a rat model. Photodiagnosis Photodyn Ther, 2010. 7(4): p. 251-7.

98. Ascencio, M., et al., Comparison of continuous and fractionated illumination during hexaminolaevulinate-photodynamic therapy. Photodiagnosis Photodyn Ther, 2008. 5(3): p. 210-6.

99. Curnow, A., et al., Light dose fractionation to enhance photodynamic therapy using 5aminolevulinic acid in the normal rat colon. Photochem Photobiol, 1999. 69(1): p. 71-6.

100. Curnow, A., J.C. Haller, and S.G. Bown, Oxygen monitoring during 5-aminolaevulinic acid induced photodynamic therapy in normal rat colon. Comparison of continuous and fractionated light regimes. J Photochem Photobiol B, 2000. 58(2-3): p. 149-55.

101. Curnow, A., A.J. MacRobert, and S.G. Bown, Comparing and combining light dose fractionation and iron chelation to enhance experimental photodynamic therapy with aminolevulinic acid. Lasers Surg Med, 2006. 38(4): p. 325-31.

102. Tudge, S.H., A.H. Kaye, and J.S. Hill, Modulation of light delivery in photodynamic therapy of brain tumors. J Clin Neurosci, 1999. 6(3): p. 227-32.

103. Linuma, S., et al., In vivo fluence rate and fractionation effects on tumor response and photobleaching: photodynamic therapy with two photosensitizers in an orthotopic rat tumor model. Cancer Res, 1999. 59(24): p. 6164-70.

104. Chen, Q., et al., The effect of light fluence rate in photodynamic therapy of normal rat brain. Radiat Res, 1992. 132(1): p. 120-3.

105. Hua, Z., et al., Effectiveness of delta-aminolevulinic acid-induced protoporphyrin as a photosensitizer for photodynamic therapy in vivo. Cancer Res, 1995. 55(8): p. 1723-31.

106. Foster, T.H., et al., Oxygen consumption and diffusion effects in photodynamic therapy. Radiat Res, 1991. 126(3): p. 296-303.

107. Daumas-Duport, C., et al., Grading of astrocytomas. A simple and reproducible method. Cancer, 1988. 62(10): p. 2152-65.

108. Al-Waili, N.S. and G.J. Butler, Phototherapy and malignancy: possible enhancement by iron administration and hyperbaric oxygen. Med Hypotheses, 2006. 67(5): p. 1148-58.

109. Chen, Q., et al., Improvement of tumor response by manipulation of tumor oxygenation during photodynamic therapy. Photochem Photobiol, 2002. 76(2): p. 197-203.

110. Huang, Z., et al., Hyperoxygenation enhances the tumor cell killing of photofrin-mediated photodynamic therapy. Photochem Photobiol, 2003. 78(5): p. 496-502.

111. Wu, S.M., et al., Protoporphyrin IX production and its photodynamic effects on glioma cells, neuroblastoma cells and normal cerebellar granule cells in vitro with 5-aminolevulinic acid and its hexylester. Cancer Lett, 2003. 200(2): p. 123-31.

112. Hirschberg, H., et al., ALA- and ALA-ester-mediated photodynamic therapy of human glioma spheroids. J Neurooncol, 2002. 57(1): p. 1-7.

113. Rachinger, W., Untersuchungen zur Porphyrin-Akkumulation im perifokalen Gliomgewebe und normalen Hirngewebe nach Gabe von 5- Aminolävulinsäure., 2006, Fakultät der LudwigMaximilians-Universität zu München, Munich, Germany.

114. Bovis, M.J., et al., Improved in vivo delivery of $m$-THPC via pegylated liposomes for use in photodynamic therapy. J Control Release, 2012. 157(2): p. 196-205. 
115. Teng, L., et al., Silencing of ferrochelatase enhances 5-aminolevulinic acid-based fluorescence and photodynamic therapy efficacy. Br J Cancer, 2011. 104(5): p. 798-807.

116. Blake, E. and A. Curnow, The hydroxypyridinone iron chelator CP94 can enhance PpIX-induced PDT of cultured human glioma cells. Photochem Photobiol, 2010. 86(5): p. 1154-60.

117. Grabb, P.A. and M.R. Gilbert, Neoplastic and pharmacological influence on the permeability of an in vitro blood-brain barrier. J Neurosurg, 1995. 82(6): p. 1053-8.

118. Hefti, M., I. Albert, and V. Luginbuehl, Phenytoin reduces 5-aminolevulinic acid-induced protoporphyrin IX accumulation in malignant glioma cells. J Neurooncol, 2012. 108(3): p. 44350 .

119. Peng, Q., et al., Antitumor effect of 5-aminolevulinic acid-mediated photodynamic therapy can be enhanced by the use of a low dose of photofrin in human tumor xenografts. Cancer Res, 2001. 61(15): p. 5824-32.

120. Linuma, S., et al., A mechanistic study of cellular photodestruction with 5-aminolaevulinic acidinduced porphyrin. Br J Cancer, 1994. 70(1): p. 21-8.

121. Peng, Q., J. Moan, and J.M. Nesland, Correlation of subcellular and intratumoral photosensitizer localization with ultrastructural features after photodynamic therapy. Ultrastruct Pathol, 1996. 20(2): p. 109-129.

122. Peng, Q., et al., Localization of fluorescent Photofrin II and aluminum phthalocyanine tetrasulfonate in transplanted human malignant tumor LOX and normal tissues of nude mice using highly light-sensitive video intensification microscopy. Int J Cancer, 1990. 45(5): p. 972-9.

123. Fingar, V.H., et al., The role of microvascular damage in photodynamic therapy: the effect of treatment on vessel constriction, permeability, and leukocyte adhesion. Cancer Res, 1992. 52(18): p. 4914-21.

124. Zhang, X., et al., Low-dose photodynamic therapy increases endothelial cell proliferation and VEGF expression in nude mice brain. Lasers Med Sci, 2005. 20(2): p. 74-9.

125. Ji, Z., et al., Induction of hypoxia-inducible factor-1alpha overexpression by cobalt chloride enhances cellular resistance to photodynamic therapy. Cancer Lett, 2006. 244(2): p. 182-9.

126. Jiang, F., et al., Angiogenesis induced by photodynamic therapy in normal rat brains. Photochem Photobiol, 2004. 79(6): p. 494-8.

127. Jiang, F., et al., Combination therapy with antiangiogenic treatment and photodynamic therapy for the nude mouse bearing U87 glioblastoma. Photochem Photobiol, 2008. 84(1): p. 128-37.

128. Coupienne, I., et al., NF-kappaB inhibition improves the sensitivity of human glioblastoma cells to 5-aminolevulinic acid-based photodynamic therapy. Biochem Pharmacol, 2010. 81(5): p. 606-16.

129. Korbelik, M., J. Sun, and I. Cecic, Photodynamic therapy-induced cell surface expression and release of heat shock proteins: relevance for tumor response. Cancer Res, 2005. 65(3): p. 101826.

130. Korbelik, M. and J. Sun, Photodynamic therapy-generated vaccine for cancer therapy. Cancer Immunol Immunother, 2006. 55(8): p. 900-9.

131. Shixiang, Y., et al., Antitumor efficacy of a photodynamic therapy-generated dendritic cell glioma vaccine. Med Oncol, 2010. 28 Suppl 1: p. S453-61.

132. Hirschberg, H., et al., Disruption of the blood-brain barrier following ALA-mediated photodynamic therapy. Lasers Surg Med, 2008. 40(8): p. 535-42.

133. Zheng, X., et al., Sensitization of cerebral tissue in nude mice with photodynamic therapy induces ADAM17/TACE and promotes glioma cell invasion. Cancer Lett, 2008. 265(2): p. 177-87.

134. Wilson, B.C., M.S. Patterson, and L. Lilge, Implicit and explicit dosimetry in photodynamic therapy: a New paradigm. Lasers Med Sci, 1997. 12(3): p. 182-99. 
135. Khan, N., et al., Repeated assessment of orthotopic glioma pO(2) by multi-site EPR oximetry: a technique with the potential to guide therapeutic optimization by repeated measurements of oxygen. J Neurosci Methods, 2011. 204(1): p. 111-7.

136. Muller, P.J. and B.C. Wilson, Photodynamic therapy: cavitary photoillumination of malignant cerebral tumours using a laser coupled inflatable balloon. Can J Neurol Sci, 1985. 12(4): p. 3713.

Table 1: Properties of the most common PS

\begin{tabular}{|c|c|c|c|c|c|c|}
\hline PS & Ref & Trade name & $\begin{array}{l}\text { Excitation } \\
(\mathrm{nm})\end{array}$ & $\begin{array}{l}\text { Drug-light } \\
\text { interval }\end{array}$ & $\begin{array}{l}\text { Clearance } \\
\text { time }\end{array}$ & Side effects \\
\hline $\begin{array}{l}\text { Porfimer } \\
\text { sodium (more } \\
\text { purified form } \\
\text { of } \mathrm{HpD} \text { ) }\end{array}$ & $12-13$ & Photofrin $\AA$ & 630 & $48-150 \mathrm{H}$ & 4-6 WEEKS & $\begin{array}{l}\text { Skin sensitization for } \\
\text { several weeks, } \\
\text { Poor tumour selectivity }\end{array}$ \\
\hline ALA-PpIX & 6,32 & $\begin{array}{l}\text { Levulan } \AA \\
\text { Gliolan } \AA\end{array}$ & $405 / 635$ & $4-8 \mathrm{H}$ & 2 DAYS & $\begin{array}{l}\text { Skin sensitization for } \\
\text { few days, } \\
\text { Nausea, Abnormal liver } \\
\text { function tests, anemia }\end{array}$ \\
\hline $\begin{array}{l}\text { BOPP } \\
\text { (boronated } \\
\text { porphyrin) }\end{array}$ & $23-25$ & & 630 & $24 \mathrm{H}$ & 4-6 WEEKS & $\begin{array}{l}\text { Skin sensitization for } \\
\text { several weeks, } \\
\text { Thrombopenia (high } \\
\text { dose) }\end{array}$ \\
\hline $\begin{array}{l}\text { Talaporfin } \\
\text { sodium }\end{array}$ & 26 & & 664 & $24 \mathrm{H}$ & 15 DAYS & $\begin{array}{l}\text { Skin sensitization for } 2 \\
\text { weeks }\end{array}$ \\
\hline mTHPC & 23 & Foscan (囚) & 652 & $48-110 \mathrm{H}$ & 15 DAYS & $\begin{array}{l}\text { Skin sensitization for } \\
\text { few weeks, } \\
\text { Hemorrhage, Pain in the } \\
\text { site of injection }\end{array}$ \\
\hline
\end{tabular}


Table 2: Histological features of some animal models of glioma [52] compared with human glioblastoma features

\begin{tabular}{|c|c|c|c|c|}
\hline & Human & Dog & U87 & U251/CNS1 \\
\hline Necrosis/Hemorrhage & + & + & - & + \\
\hline Pseudopallisading & + & + & - & + \\
\hline Angiogenesis & + & + & + & + \\
\hline Endothelial proliferation & + & + & - & $+/-$ \\
\hline Invasion & + & + & $+/-$ & + \\
\hline
\end{tabular}

